\title{
A brief report on combination chemotherapy and anti-programmed death (ligand) 1 treatment in small-cell lung cancer: Did we choose the optimal chemotherapy backbone?
}

\author{
Joanne M. Mankor ${ }^{\mathrm{a}, 1}$, Fenneke Zwierenga ${ }^{\mathrm{a}, 1}$, Daphne W. Dumoulin ${ }^{\text {a }}$, \\ Jacques J.C. Neefjes ${ }^{b}$, Joachim G.J.V. Aerts ${ }^{a, *}$ \\ a Department of Pulmonary Medicine, Erasmus MC, Rotterdam, the Netherlands \\ ${ }^{\mathrm{b}}$ Department of Cell and Chemical Biology, Leiden University Medical Centre, Leiden, the Netherlands
}

Received 24 March 2020; received in revised form 8 June 2020; accepted 21 June 2020

\section{KEYWORDS \\ Small-cell lung cancer; Immunotherapy; Immunologic factors; Etoposide; Calreticulin; Immunogenic cell death}

\begin{abstract}
Extensive-stage small-cell lung cancer (ES-SCLC) is an aggressive cancer that remains very hard to treat. The life expectancy of a patient diagnosed with this disease has not changed over the past three decades. Recently, three large clinical studies showed a survival benefit by adding an anti-programmed death (ligand) 1 (PD-(L)1 antibody to the current chemotherapy regimen. Although significant and important, the benefit seems less than what has been achieved in patients with non-small-cell lung cancer treated with chemoimmunotherapy. A number of hypotheses have been explored to explain this discrepancy. Here, we hypothesise that the current chemotherapy backbone in ES-SCLC does not contain the optimal drugs to trigger immunogenic cell death and therefore does not induce a synergy between chemotherapy and immune checkpoint inhibitor therapy. Thereby, we advocate that doxorubicin treatment instead of etoposide should be reconsidered as standard-of-care (SoC) first-line treatment of SCLC.

(C) 2020 Published by Elsevier Ltd.
\end{abstract}

\footnotetext{
* Corresponding author: Erasmus MC, Dr. Molewaterplein 40, 3015, GD, Rotterdam, the Netherlands. E-mail address: j.aerts@erasmusmc.nl (J.G.J.V. Aerts).

1 These authors contributed equally.
} 
Small-cell lung cancer (SCLC) is an aggressive type of cancer. At diagnosis, approximately two-third of the patients are diagnosed with extensive-stage (ES) disease. ES-SCLC treatment options remain limited, resulting in a poor prognosis that did not improve in the past three decades. For many years, the standard-of-care (SoC) treatment regimen most used for patients with ES-SCLC consists of 4-6 cycles of platinum-based chemotherapy (cisplatin/carboplatin) and etoposide. ES-SCLC responds well to chemotherapy, but recurrence of disease develops rapidly [1].

The treatment landscape of thoracic malignancies in general changed dramatically in the past decades, due to the discovery of immune checkpoint inhibitor (ICI) therapy, i.e. programmed death 1 (PD-1), programmed death ligand 1 (PD-L1) and cytotoxic T-lymphocyte-associated antigen 4 (CTLA-4) monoclonal antibodies [2]. Nonetheless, it should be noted that within the thoracic malignancies mainly patients with non-smallcell lung cancer (NSCLC) benefit from this discovery [3]. However, two important factors hinted towards a beneficial role for ICI in ES-SCLC. First, the response to chemotherapy, which is seen in the majority of patients with ES-SCLC, induces a reduction in tumour burden and thus potentially in the immunosuppressive environment created by the tumour, which is beneficial for ICI response [4]. Second, the high tumour mutational burden (TMB) described in ES-SCLC, potentially resulting in a large number of neoantigens, has been shown to be a promising predictive biomarker of ICI efficacy in several types of cancer. Although the predictive value of TMB appeared to be limited in prospective clinical studies, high TMB in ES-SCLC did raise hope for similar ICI clinical responses in SCLC and NSCLC tumours [5].

Various clinical trials investigated checkpoint blockade in ES-SCLC. Results of single-agent checkpoint inhibitor trials in the second-line or later setting, have been disappointing as benefit was limited, in contrast to the results seen in NSCLC. Anti-PD-1 antibody treatment, with or without the addition of anti-CTLA, has been explored in the single arm trials of Checkmate 032 [6] KEYNOTE-028 [7] and KEYNOTE158 [8]. The pooled analysis of KEYNOTE-028 and KEYNOTE-158 reported a response rate of $19,3 \%$, (95\% confidence interval [CI]: 11.4-29.4), a median progression-free survival (PFS) of 2.0 months $(95 \% \mathrm{CI}$ : 1.9-3.4) and a median overall survival (OS) of 7.7 months (95\% CI: 5.2-10.1) [9]. Checkmate 331 [10] and IFCT-1603 [11] compared SoC chemotherapy to nivolumab and atezolizumab, respectively, but both failed to improve OS in patients with SCLC requiring second-line treatment. Furthermore, single-agent anti-PD-1 treatment in third-line setting has been investigated in singlearm trials and US Food and Drug Administration approval was granted based on objective response rates of only $10-20 \%$. In NSCLC in contrast, higher number of responders and more durable responses to singleagent ICI are seen [12-14].

The high sensitivity to chemotherapy that characterises most SCLCs, results in massive tumour antigen release from dying tumour cells, which theoretically renders these tumours as sensitive to ICI as NSCLC tumours. A number of phase III clinical trials investigated this strategy. The first phase III clinical trial that investigated ICI-therapy in combination with first-line therapy, studied the role of an anti-CTLA-4 antibody in combination with platinum and etoposide. No difference was established in PFS nor OS [15]. Furthermore, the IMpower 133 phase III randomised trial evaluated the efficacy and safety of atezolizumab (anti-PD-L1) with carboplatin and -etoposide. The study showed a significant improvement in PFS (hazard ratio for disease progression or death: $0.77 ; 95 \% \mathrm{CI}: 0.62-0.96 ; \mathrm{P}=0.02$ ) and OS (hazard ratio for death: $0.70 ; 95 \%$ CI: 0.54 to $0.91 ; \mathrm{p}=0.007)$, but response rates did not differ between the two arms [16]. The phase III, randomised CASPIAN trial assessed first-line durvalumab (anti-PD-L1) and etoposide with either cisplatin or carboplatin versus platinum-etoposide alone. This resulted in significantly longer OS (HR for disease progression or death: $0.73 ; 95 \%$ CI: $0.59-0.91 ; \mathrm{P}=0.0047$ ). Significance of PFS could not be tested due to the study design, but median PFS was 5.1 in the combination treatment arm versus 5.4 months in the platinumetoposide alone arm, resulting in a HR of $0.78(95 \% \mathrm{CI}$ : 0.65-0.94) [17]. The KEYNOTE-604 phase III randomised trial evaluated the addition of pembrolizumab (anti-PD-1) to etoposide-platinum (either cisplatin or carboplatin) versus placebo/etoposide-platinum. A prior interim analysis demonstrated a significant improvement in PFS (HR for disease progression: $0.75 ; 95 \% \mathrm{CI}$ : 0.61-0.91). Although OS did improve as well, the OS results did not meet the criteria for statistical significance per the pre-specified statistical plan (HR for death: 0.80; 95\% CI: 0.64-0.98) [18]. In conclusion, the clinical trials summarised here demonstrated significant differences in favour of the chemotherapy ICI combination treatment arm, but only a minority of patients with ES-SCLC seems to benefit from ICI in combination with chemotherapy. No biomarker is yet to be found to identify this minority, partly due to the confounding nature of the chemosensitive SCLC tumours. In NSCLC, the benefit from chemotherapy ICI combination treatment compared with chemotherapy only, is much more pronounced. KEYNOTE-189 investigated SoC chemotherapy plus pembrolizumab versus SoC chemotherapy plus placebo in NSCLC and found a hazard ratio for progression or death of $0.52(95 \% \mathrm{CI}: 0.43$ to $0.64 ; \mathrm{P}<0.001)$ and 12 -month OS rate of $61.7 \%$ vs. $52.2 \%$ (hazard ratio for death: $0.59 ; 95 \%$ CI: 0.38 to 0.92 ) in the pembrolizumab vs. placebo groups [19]. Currently, there is also in NSCLC no biomarker available to predict improved outcome on combination treatment. 
Clearly, there is a discrepancy between NSCLC and SCLC clinical responses to chemotherapy ICI combination treatment. SCLC tends to be intrinsically more resistant to ICI than NSCLC, and different mechanisms of resistance are likely to be in place. In NSCLC, benefits in terms of response rates, PFS and OS are potentially due to synergistic effects of the two treatment modalities. It stands out that in SCLC combination trials, a clear separation of the PFS and OS curves can only be seen after 4-7 months. Late separation of the curves, in addition to the lack of improvement in response rates, do support the absence of a synergistic effect between the two treatment modalities. In this brief report, we explore the optimal chemotherapy backbone for ICI combination treatment in SCLC, aiming for true synergy.

Immunogenic cell death (ICD) is a key mechanism in the process of immune modulation by cytotoxic chemotherapies. ICD results in regulated activation of an immune response, in the absence of 'pathogen-associated molecule patterns'. In contrast, 'damage-associated molecular patterns' (DAMPs), molecules that are expressed or released upon cellular stress responses or cell death, can exert powerful immunogenic signals by binding to pattern recognition receptors on immune cells. These signals activate a cascade, resulting in the activation of cytotoxic CD8 T cells that can eliminate tumour cells. So far, 4 key DAMPs have been recognised to play an important role in chemotherapyinduced ICD [20]. First, the release of the nuclear protein non-histone chromatin-binding protein high-mobility group box 1 (HMGB1) into the extracellular space can activate Toll-like receptor 4 that is expressed on dendritic cells (DCs). Second, type I interferon signalling by dying cancer cells can upregulate chemotactic factors on surrounding cells that help attract $T$ cells to the tumour site. Third, if apoptotic cell death is preceded by autophagy, ATP can be released and attract myeloid cells. And finally, the ER protein calreticulin (CALR) translocates to the cell membrane in response to the induction of endoplasmatic reticulum (ER) stress, and provides an important 'eat-me' signal to antigen-presenting cells, by interacting with CD91 on the engulfing cell [21]. CALR appears to play a vital role

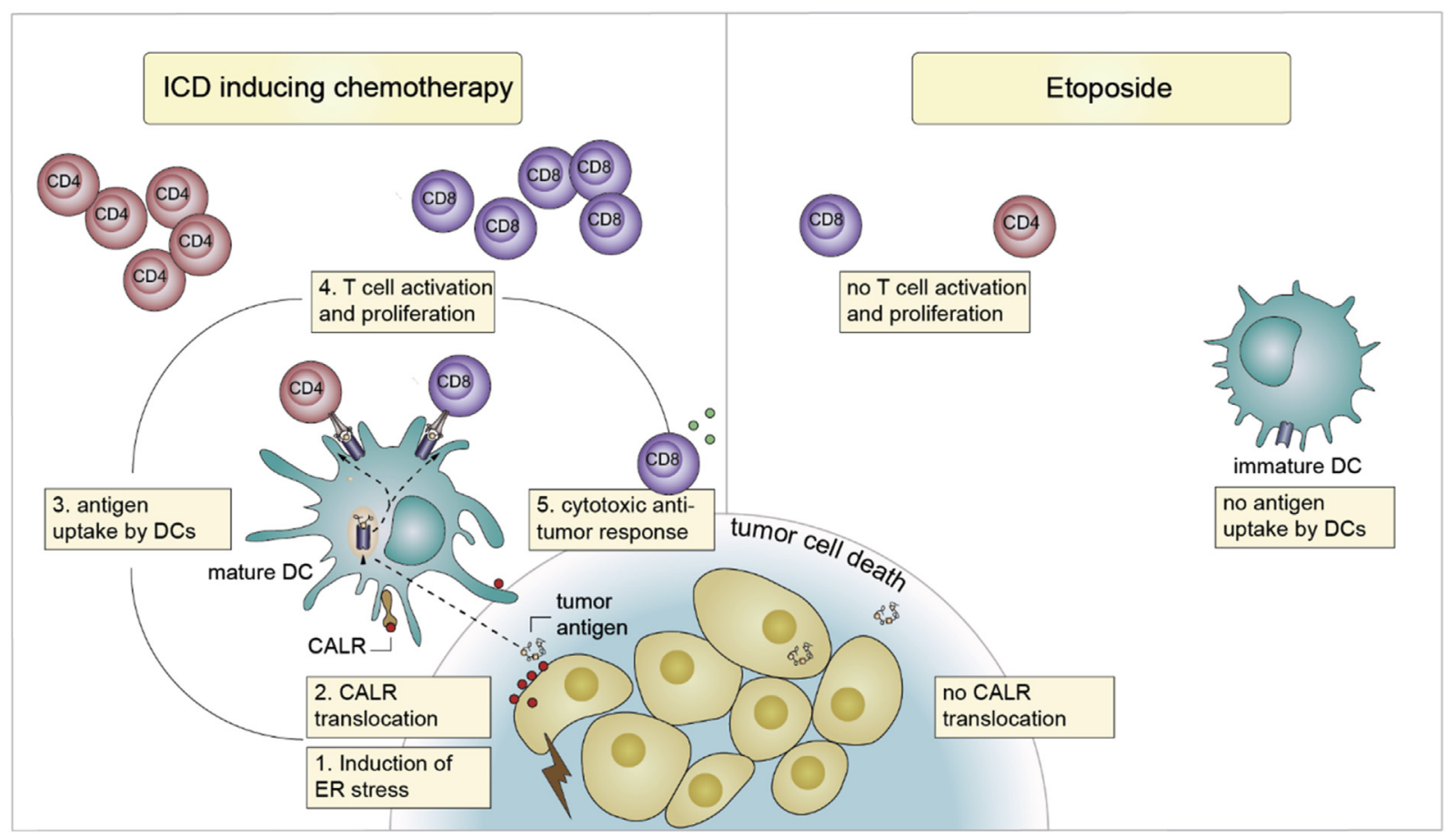

Fig. 1. Differences in the immunological response to dying tumour cells that is initiated by immunogenic cell death (ICD)-inducing chemotherapies and etoposide. The left panel of this figure depicts how ICD inducing chemotherapies can promote a cytotoxic anti-tumour immune response by CD8 T cells. First (1), chemotherapy can induce endoplasmatic reticulum (ER) stress in tumour cells. In response to ER stress, the ER protein calreticulin (CALR) translocates to the tumour cell surface. CALR functions as a damage-associated molecular pattern (DAMP), and this damage signal stimulates DCs to take up tumour antigens (3). In response, DCs will mature and prime CD4 and CD8 T cells by displaying the appropriate peptide-MHC ligand, which promotes T cell activation and proliferation (4). Priming of naive CD8 T cells generates cytotoxic effector CD8 T cells that are capable of direct tumour cell killing (5). In contrast, the right panel of this figure depicts the lack of T cell activation during etoposide treatment. Because etoposide is not a potent ICD inducer, CALR is absent on the tumour cell surface, thereby diminishing activation and maturation of DCs. These DCs are less likely to take up tumour antigens and to activate CD4 and CD8 T cells. As a result, T cell activation and proliferation does not occur and a cytotoxic anti-tumour response is not initiated. CALR = calreticulin, ER stress = endoplasmatic reticulum stress, DC = dendritic cell, ICD = immunogenic cell death. 
in the context of ICD, induced by chemotherapeutic agents.

Obeid et al. [22] performed a number of in vitro and in vivo experiments and concluded that CALR exposure was necessary for successful ICD. First, they found that anthracyclins are highly efficient ICD inducers and that the immunogenicity of anthracyclins could be abrogated by the blockade or knockdown of CALR, which in turn suppressed phagocytosis of dying tumour cells by DCs. On the other hand, CALR translocation was lacking in etoposide-treated mice and administration of recombinant CALR could restore ICD and enhance antitumour effects in this treatment regimen. Bezu et al. [20] supported these findings in their review and concluded that, even though etoposide does regulate ATP secretion and HMGB1 release, CALR translocation was inevitable for successful ICD induction. In conclusion, the lack of CALR translocation may be a key feature that is missing in patients treated with platinum-etoposide and ICI (Fig. 1).

Until 2000, anthracyclin-based chemotherapy in the form of doxorubicin was used in Europe as the $\mathrm{SoC}$ regimen for first-line treatment in SCLC [23]. Doxorubicin and etoposide share the same molecular targets and induce DNA double-stranded breaks in an almost identical manner. Clinical benefit of platinum-etoposide and doxorubicin regimens was shown to be similar in several trials. A meta-analysis of cisplatin containing regimens versus regimens without platinum however, put doxorubicin to the second place of SCLC treatment [24]. Now, in the light of ICD induction, doxorubicin treatment for SCLC should be brought back under consideration.

Because the current regimen consists of etoposide combined with cisplatin or carboplatin, it is inevitable in this context to also consider the immunogenic capacities of platinum agents. It is important to notice that chemically related chemotherapeutic agents can have entirely different capacities to trigger ICD. Preclinical studies showed that cisplatin is intrinsically incapable of inducing ICD, due to the lack of ER stress-dependent CALR translocation [25]. Carboplatin induces cell death in a similar manner to cisplatin [26]. Oxaliplatin on the contrary, is known to be a powerful ICD inducer. However, oxaliplatin has not been proved to be effective in SCLC and thus the platinum chemotherapeutics appear not to be the right agents to combine with ICI in the context of SCLC.

It should be mentioned, that etoposide combination treatment was also one of the potential agents used in the PACIFIC trial. This trial investigated the benefit of adding durvalumab as maintenance treatment in stage III NSCLC [27]. Approximately one quarter of patients received etoposide, and up to this date, no data are present on differences in efficacy of the chemotherapy arms. But one should realise that these patients were irradiated concurrently and radiotherapy is known to be a potent ICD inducer. This may have reduced the need for DAMP release induced by chemotherapy.

In conclusion, although exciting new treatment options are developed by combining chemotherapy and ICI, the combination should be designed with care. By adding chemotherapy to ICI, we should aim to reinforce tumour immunogenicity and alleviate immunosuppression. Therefore, we argue that a systematic investigation of ICD inducing capacities of currently available chemotherapies for SCLC is urgently needed. This knowledge should be the basis for further clinical investigations.

\section{Conflict of interest statement}

J.G.J.V.A reports being a member of the advisory board for and/or received speakers fee from Eli Lilly, Roche, Boehringer Ingelheim, BMS, AstraZeneca and MSD; is a stock owner of Amphera B.V. Immunotherapy; has a patent pending on tumour lysate antigen. D.W.D. has received speakers fee from Roche, Novartis, BMS, MSD, AstraZeneca and Pfizer. All the remaining authors have declared no conflicts of interest.

\section{References}

[1] Früh M, de Ruysscher D, Popat S, Crino L, Peters S, Felip E. Smallcell lung cancer (SCLC): ESMO Clinical Practice Guidelines for diagnosis, treatment and follow-up †. ESMO Updated Clin Pract Guideline 2013;24:vi99-105. https://doi.org/10.1093/annonc/mdt178.

[2] Ribas A, Wolchok JD. Cancer immunotherapy using checkpoint blockade. Science 2018. https://doi.org/10.1126/science.aar4060.

[3] Proto C, Ferrara R, Signorelli D, Lo Russo G, Galli G, Imbimbo M, et al. Choosing wisely first line immunotherapy in non-small cell lung cancer (NSCLC): what to add and what to leave out. Canc Treat Rev 2019;75:39-51. https: //doi.org/10.1016/j.ctrv.2019.03.004.

[4] Huang AC, Postow MA, Orlowski RJ, Mick R, Bengsch B, Manne $\mathrm{S}$, et al. T-cell invigoration to tumour burden ratio associated with anti-PD-1 response. Nature 2017. https: //doi.org/10.1038/nature22079.

[5] Samstein RM, Lee C-H, Shoushtari AN, Hellman MD, Shen R, Janjigian YY, et al. Tumor mutational load predicts survival after immunotherapy across multiple cancer types. Nat Gen January 2019;1. https://doi.org/10.1038/s41588-018-0312-8.

[6] Antonia SJ, López-Martin JA, Bendell J, Ott PA, Taylor M, Eder JP, et al. Nivolumab alone and nivolumab plus ipilimumab in recurrent small-cell lung cancer (CheckMate 032): a multicentre, open-label, phase 1/2 trial. Lancet Oncol 2016;17(7): 883-95. https://doi.org/10.1016/S1470-2045(16)30098-5.

[7] Ott PA, Elez E, Hiret S, Kim D, Morosky A, Saraf S, et al. Pembrolizumab in patients with extensive-stage small-cell lung cancer: results from the phase Ib KEYNOTE-028 study. J Clin Oncol 2017; 35(34):3823-9. https://doi.org/10.1200/jco.2017.72.5069.

[8] Chung HC, Lopez-Martin JA, Kao SC-H, Miller WH, Ros W, Gao B, et al. Phase 2 study of pembrolizumab in advanced smallcell lung cancer (SCLC): KEYNOTE-158. J Clin Oncol 2018; 36(15_suppl). https://doi.org/10.1200/jco.2018.36.15_suppl.8506. 8506-8506.

[9] Chung HC, Piha-Paul SA, Lopez-Martin J, Krishnan S, Norwood K, Ott PA, et al. Pembrolizumab after two or more lines of previous therapy in patients with recurrent or metastatic SCLC: results from the KEYNOTE-028 and KEYNOTE-158 
studies. J Thorac Oncol 2020;15(4):618-27. https: //doi.org/10.1016/j.jtho.2019.12.109.

[10] Reck M, Vicente D, Ciuleanu T, Gettinger S, Peters S, Horn L, et al. LBA5 Efficacy and safety of nivolumab (nivo) monotherapy versus chemotherapy (chemo) in recurrent small cell lung cancer (SCLC): results from CheckMate 331. Abstract Book of ESMO Immuno-Oncology Congress 2018 13-16 December, Geneva, Switzerland Ann Oncol 2018;29:x39-43. https: //doi.org/10.1093/annonc/mdy511.

[11] Pujol JL, Greillier L, Audigier-Valette C, Langlais A, Morin F, Souquet P-J, et al. A randomized non-comparative phase II study of anti-programmed cell death-ligand 1 atezolizumab or chemotherapy as second-line therapy in patients with small cell lung cancer: results from the IFCT-1603 trial. J Thorac Oncol 2019; 14(5):903-13. https://doi.org/10.1016/j.jtho.2019.01.008.

[12] Brahmer J, Reckamp KL, Baas P, Crino L, Eberhardt WEE, Poddubskaya E, et al. Nivolumab versus docetaxel in advanced squamous-cell non-small-cell lung cancer. N Engl J Med 2015; 373(2):123-35. https://doi.org/10.1056/NEJMoa1504627.

[13] Borghaei H, Paz-Ares L, Horn L, Spigel DR, Steins M, Ready NE, et al. Nivolumab versus docetaxel in advanced nonsquamous non-small-cell lung cancer. N Engl J Med 2015; 373(17):1627-39. https://doi.org/10.1056/NEJMoa1507643.

[14] Herbst RS, Baas P, Kim DW, Kim D-W, Enriqueta F, PérezGracia JL, et al. Pembrolizumab versus docetaxel for previously treated, PD-L1-positive, advanced non-small-cell lung cancer (KEYNOTE-010): a randomised controlled trial. Lancet 2016; 387(10027):1540-50. https://doi.org/10.1016/S0140-6736(15)01281-7.

[15] Reck M, Luft A, Szczesna A, Havel L, Kim S-W, Akerley W, et al. Phase III randomized trial of ipilimumab plus etoposide and platinum versus placebo plus etoposide and platinum in extensivestage small-cell lung cancer. J Clin Oncol 2016;34(31):3740-8. https://doi.org/10.1200/JCO.2016.67.6601.

[16] Horn L, Mansfield AS, Szczęsna A, Krzakowski M, Hochmair MJ, Huemer F, et al. First-line atezolizumab plus chemotherapy in extensive-stage small-cell lung cancer. N Engl J Med 2018;379(23): 2220-9. https://doi.org/10.1056/NEJMoa1809064.

[17] Paz-Ares L, Dvorkin M, Chen Y, Reinmuth N, Hotta K, Trukhin D, et al. Durvalumab plus platinum-etoposide versus platinum-etoposide in first-line treatment of extensive-stage small-cell lung cancer (CASPIAN): a randomised, controlled, open-label, phase 3 trial. Lancet October 2019. https: //doi.org/10.1016/s0140-6736(19)32222-6.

[18] Merck \& Co., Inc. - Merck's KEYTRUDA ${ }^{\circledR}$ (pembrolizumab) in Combination with Chemotherapy Significantly Improved
Progression-Free Survival Compared to Chemotherapy Alone as First-Line Treatment for Extensive Stage Small Cell Lung Cancer. https://investors.merck.com/news/press-release-details/2020/ Mercks-KEYTRUDA-pembrolizumab-in-Combination-withChemotherapy-Significantly-Improved-Progression-FreeSurvival-Compared-to-Chemotherapy-Alone-as-First-LineTreatment-for-Extensive-Stage-Small-Cell-Lung-Cancer/default. aspx. Accessed July 24, 2020.

[19] Gandhi L, Rodríguez-Abreu D, Gadgeel S, Esteban E, Felip E, De Angelis F, et al. Pembrolizumab plus chemotherapy in metastatic non-small-cell lung cancer. N Engl J Med 2018;378(22): 2078-92. https://doi.org/10.1056/NEJMoa1801005.

[20] Bezu L, Gomes-da-Silva LC, Dewitte H, Breckpot K, Fucikova J, Spisek R, et al. Combinatorial strategies for the induction of immunogenic cell death. Front Immunol 2015;6(APR). https: //doi.org/10.3389/fimmu.2015.00187.

[21] Garg AD, Krysko Dv, Verfaillie T, Kaczmarek A, Ferreira GB, Marysael T, et al. A novel pathway combining calreticulin exposure and ATP secretion in immunogenic cancer cell death. EMBO J 2012; 31(5):1062-79. https://doi.org/10.1038/emboj.2011.497.

[22] Obeid M, Tesniere A, Ghiringhelli F, Fimia GM, Apetoh L, Perfettini J-L, et al. Calreticulin exposure dictates the immunogenicity of cancer cell death. Nat Med 2007;13(1):54-61. https: //doi.org/10.1038/nm1523.

[23] Chan BA, Coward JIG. Chemotherapy advances in small-cell lung cancer. J Thorac Dis 2013;5(SUPPL.5). https: //doi.org/10.3978/j.issn.2072-1439.2013.07.43.

[24] Pujol JL, Carestia, Daurès JP. Is there a case for cisplatin in the treatment of small-cell lung cancer? A meta-analysis of randomized trials of a cisplatin-containing regimen versus a regimen without this alkylating agent. Br J Canc 2000;83(1):8-15. https: //doi.org/10.1054/bjoc.2000.1164.

[25] Martins I, Kepp O, Schlemmer F, Adjemian S, Tailler M, Shen S, et al. Restoration of the immunogenicity of cisplatin-induced cancer cell death by endoplasmic reticulum stress. Oncogene 2011; 30(10):1147-58. https://doi.org/10.1038/onc.2010.500.

[26] Galluzzi L, Buqué A, Kepp O, Zitvogel L, Kroemer G. Immunological effects of conventional chemotherapy and targeted anticancer agents. Canc Cell 2015;28(6):690-714. https: //doi.org/10.1016/j.ccell.2015.10.012.

[27] Antonia SJ, Villegas A, Daniel D, Vicente D, Murakami S, Hui R, et al. Durvalumab after chemoradiotherapy in stage III non-small-cell lung cancer. N Engl J Med 2017;377(20):1919-29. https://doi.org/10.1056/NEJMoa1709937. 